\title{
Central airways remodeling in COPD patients
}

This article was published in the following Dove Press journal:

International Journal of COPD

I September 2014

Number of times this article has been viewed

Laura Pini'

Valentina Pinelli²

Denise Modina'

Michela Bezzi ${ }^{3}$

Laura Tiberio ${ }^{4}$

Claudio Tantucci'

'Unit of Respiratory Medicine,

Department of Clinical and

Experimental Sciences, University of Brescia, ${ }^{2}$ Department of Respiratory Medicine, Spedali Civili di Brescia,

${ }^{3}$ Department Bronchoscopy, Spedali

Civili di Brescia, ${ }^{4}$ Department of

Molecular and Translational Medicine,

University of Brescia, Brescia, Italy
Correspondence: Laura Pini

Unit of Respiratory Medicine,

Department of Clinical and Experimental

Sciences, University of Brescia,

Piazzale Spedali Civili, I, 25I 23

Brescia, Italy

Tel +39303996263

Email laura.pini@unibs.it
Background: The contribution to airflow obstruction by the remodeling of the peripheral airways in chronic obstructive pulmonary disease (COPD) patients has been well documented, but less is known about the role played by the large airways. Few studies have investigated the presence of histopathological changes due to remodeling in the large airways of COPD patients.

Objectives: The aim of this study was to verify the presence of airway remodeling in the central airways of COPD patients, quantifying the airway smooth muscle (ASM) area and the extracellular matrix (ECM) protein deposition, both in the subepithelial region and in the ASM, and to verify the possible contribution to airflow obstruction by the above mentioned histopathological changes.

Methods: Biopsies of segmental bronchi spurs were performed in COPD patients and control smoker subjects and immunostained for collagen type I, versican, decorin, biglycan, and alpha-smooth muscle actin. ECM protein deposition was measured at both subepithelial, and ASM layers.

Results: The staining for collagen I and versican was greater in the subepithelial layer of COPD patients than in control subjects. An inverse correlation was found between collagen I in the subepithelial layer and both forced expiratory volume in 1 second and ratio between forced expiratory volume in 1 second and forced vital capacity. A statistically significant increase of the ASM area was observed in the central airways of COPD patients versus controls.

Conclusion: These findings indicate that airway remodeling also affects the large airways in COPD patients who have greater deposition of ECM proteins in the subepithelial layer and a larger smooth muscle area than control smoker subjects. These changes may contribute to chronic airflow obstruction in COPD patients.

Keywords: COPD, central airways, remodeling, airway smooth muscle, proteoglycans

\section{Introduction}

Tissue remodeling is an important feature in the airways of many patients with chronic obstructive pulmonary disease (COPD) and contributes to the progressive nature and severity of airflow obstruction.

Remodeling of the peripheral airway wall is well described and has been acknowledged as the core hallmark of chronic bronchiolitis leading to COPD. ${ }^{1,2}$ Many studies have shown that chronic inflammation is associated with airway wall thickening, alveolar detachments, and reduction of airway-parenchyma interdependence leading to luminal narrowing. ${ }^{3-6}$ Moreover, Hogg et al have shown that the progression of airflow obstruction in COPD patients is strongly associated with the thickening of the airway wall, the degree to which the lumen was filled with mucous exudates, and the amount of inflammation in the small airways. ${ }^{7}$ 
In the last years progress toward understanding the remodeling and inflammation in large airways of COPD patients has also been made. Tiddens et al documented that, in COPD patients, the portion of central airways composed by epithelial and subepithelial layers is thicker compared to those of healthy subjects, and also demonstrated a negative correlation between the thickness of this portion and forced expiratory volume in 1 second $\left(\mathrm{FEV}_{1}\right)$ /forced vital capacity (FVC) ratio. No differences were found in the smooth muscle (ASM) area of the cartilaginous airway between patients and the control group. ${ }^{8}$ More recently, Liesker et al found a thickening of the reticular basement membrane in central airways of both asthmatic and COPD patients due to different extracellular matrix (ECM) alterations. ${ }^{9}$

Furthermore, the bronchial expression pattern of various ECM components such as collagen, fibronectin and laminin $\beta_{2}$ in central airways of COPD patients was studied by Kranenburg et al. Their data showed that COPD patients have an increased bronchial deposition of ECM proteins in the subepithelial basement membrane that contributes to airway remodeling and reduced lung function. ${ }^{10}$

A reduction in type I collagen content in the inner layer, and of elastic fibers in all compartments of the large airways of COPD patients, was also found by Antoni et al. ${ }^{11}$ However, very little is known about the role played by other ECM molecules, such as proteoglycans (PGs), ${ }^{12}$ and the smooth muscle mass change in central airway remodeling in COPD patients.

In fact, increased ECM protein expression and smooth muscle layer area in central airways, by thickening the airway wall, may lead to a further increase of airway resistance and thus induce relevant functional consequences in COPD patients. Therefore, in this work, specimens obtained from endobronchial biopsies were used to evaluate the contribution of ECM molecules and smooth muscle mass to central airway wall remodeling in COPD patients.

\section{Subjects and methods Subjects}

Between January and June 2010, eight COPD patients (age range 62-82 years, five were classified as Global initiative on Obstructive Lung Disease [GOLD] stage moderate and three were classified as GOLD stage severe) and five control subjects (age range 37-84 years) were recruited from the bronchoscopy service of the Clinical Hospital of Brescia (Table 1), following approval from the Spedali Civili di Brescia's ethics committees and having obtained informed consent from all subjects to participate in the
Table I Patients' characteristics and spirometry indices

\begin{tabular}{llll}
\hline & $\begin{array}{l}\text { COPD } \\
(\mathbf{n}=\mathbf{8})\end{array}$ & $\begin{array}{l}\text { Controls } \\
(\mathbf{n}=\mathbf{5})\end{array}$ & P-value \\
\hline Sex (M/F) & $6 / 2$ & $5 / 0$ & \\
Age (years) & $7 I \pm 3$ & $58 \pm 7$ & $\mathrm{~ns}$ \\
FEV $_{1}(\%$ pred) & $70 \pm 10$ & $115 \pm 12$ & $<0.05$ \\
FEV/FVC (\%) & $55 \pm 4$ & $77 \pm 3$ & $<0.05$ \\
Smoking habit (pack/years) & $59 \pm 12$ & $5 I \pm 20$ & $\mathrm{~ns}$ \\
\hline
\end{tabular}

Note: Values are presented as mean \pm standard deviation.

Abbreviations: COPD, chronic obstructive pulmonary disease; FEV expiratory volume in the first second; \% pred, \% predicted; FVC, forced vital capacity; $M$, male; F, female; ns, not significant.

present study. Standard pulmonary function testing was performed on all subjects.

In the presence of well-known risk factors, COPD has been defined, according to the American Thoracic Society criteria, by $\mathrm{FEV}_{1} / \mathrm{FVC}$ ratio $<70 \%$ after administration of $400 \mu \mathrm{g}$ nebulized albuterol (salbutamol). ${ }^{13}$

COPD subjects were only deemed suitable for the study if they had not experienced exacerbated COPD symptoms during the 3 months preceding the commencement of the study. Exacerbation was defined as increased dyspnea associated with modification in the quality/quantity of sputum that led to the subjects seeking medical attention and/or changing their medical treatment. ${ }^{14}$

The control subjects had normal pulmonary function. None of the subjects had acute respiratory tract infections or had received glucocorticoids during the 3 months preceding bronchoscopy.

\section{Procedures}

Bronchoscopies were performed according to standard procedures. ${ }^{15} \mathrm{~A}$ total of 2 biopsy specimens per patient were taken at the carina of segmental bronchi from right upper and middle lobar bronchi, fixed in $4 \%$ paraformaldehyde and finally embedded in paraffin. Tissue slices ( $3 \mu \mathrm{m}$ thickness) were then placed on polylysine $(0.1 \%)$ coated glass slides.

\section{Immunohistochemistry}

Tissue sections were deparaffinized, rehydrated, and preincubated with goat serum for 5 minutes. Subsequently, sections were incubated with collagen type I primary antibodies (monoclonal rabbit, anti-human; Cederlane, Hornby, ON, Canada), versican (monoclonal mouse, anti-human 1 [generously provided by Developmental Studies Hybridoma Bank, Iowa City, IA, USA]), decorin (monoclonal mouse, anti-human [generously provided by Developmental Studies Hybridoma Bank]), biglycan (polyclonal rabbit, anti-human [provided 
by P Roughley, Shriners Hospital, McGill University]), and alpha-smooth muscle actin ( $\alpha$-SMA) (monoclonal mouse, anti-human; Sigma-Aldrich Co, St Louis, MO, USA).

Horseradish peroxidase-conjugated anti-rabbit or antimouse (ChemMate EnVision ${ }^{\mathrm{TM}}$ System; DakoCytomation, Troy, MI, USA) was used as a secondary antibody. Diaminobenzidine was used as the chromogen. The sections were counterstained with hematoxylin. For negative controls, the primary antibody was omitted from the staining.

\section{Morphometry}

The slides were analyzed using the Olympus light microscope BX51, (Carson Group Inc., Markam, ON, Canada) and images were examined using Image-Pro Plus software (Media Cybernetics, Silver Spring, MD, USA).

Detection of collagen I and PGs staining in the subepithelial layer was performed at $4 \times$ magnification. To define the subepithelial layer, the reticular basement membrane was identified and the analyses were extended down to the smooth muscle layer with the exclusion of blood vessels and mucous glands. Previously determined stain-specific color settings were examined using Image-Pro Plus software to calculate the area of positive staining. That area $\left(\mu \mathrm{m}^{2}\right)$ was then standardized for total subepithelial area. An average percentage of positive staining for all biopsies examined in each subject was calculated and used for statistical analysis. ${ }^{16-18}$

Positive staining of collagen type I and PGs in the smooth muscle layer was detected at 100× magnification using a $1.0 \mathrm{~mm}^{2}$ eyepiece graticule. The amount of PGs within, or overlying the smooth muscle layer was expressed as the percentage of positive staining overlapping the cross-points of a 100 point grid. Ten fields (randomly chosen) were analyzed per biopsy. Analyses were performed in the areas where the ASM was clearly identified and surrounding connective tissue was always excluded. ${ }^{16-18}$

To detect positive staining of $\alpha$-SMA, $20 \times$ magnification was used and the following measurements were taken: area of positive staining, basement membrane perimeter $\left(\mathrm{P}_{\mathrm{bm}}\right)$, and total area of smooth muscle bundles. The signal coming from blood vessels was excluded. Results were expressed as area of positive staining $\left(\mu \mathrm{m}^{2}\right)$ standardized for basement membrane length squared $\left(\mathrm{P}_{\mathrm{bm}}{ }^{2}\right) \cdot{ }^{16-18}$

\section{Statistical analysis}

Data are presented as mean \pm standard error; data were analyzed using the Mann-Whitney $U$ test, and a $P$-value of $<0.05$ was considered to be statistically significant.
The expression of ECM proteins in the subepithelial layer and the amount of $\alpha$-SMA were also correlated with $\mathrm{FEV}_{1}$ (\% predicted), and $\mathrm{FEV}_{1} / \mathrm{FVC}(\%)$ ratio using the Pearson correlation analysis.

\section{Results}

Characteristics of the subjects enrolled in the present study between January and June 2010, are shown in Table 1.

\section{Immunohistochemistry}

\section{Collagen I and PGs in the subepithelial layer}

Positive staining for collagen I and PGs was identified in the subepithelial layer of all airway specimens of both COPD patients and control subjects. Staining for collagen I and versican was significantly increased in COPD patients versus controls $(P<0.05)$, and there was only a trend toward increased decorin deposition in COPD patients, (Figure 1). No differences were detected between the immunostaining for biglycan in the COPD and control groups (data not shown). Measurements of collagen I, versican, and decorin in the subepithelial area are shown in Figure 2.

\section{Airway smooth muscle}

The ASM was identified by immunostaining with $\alpha$-SMA antibody. There was a statistically significant increase in
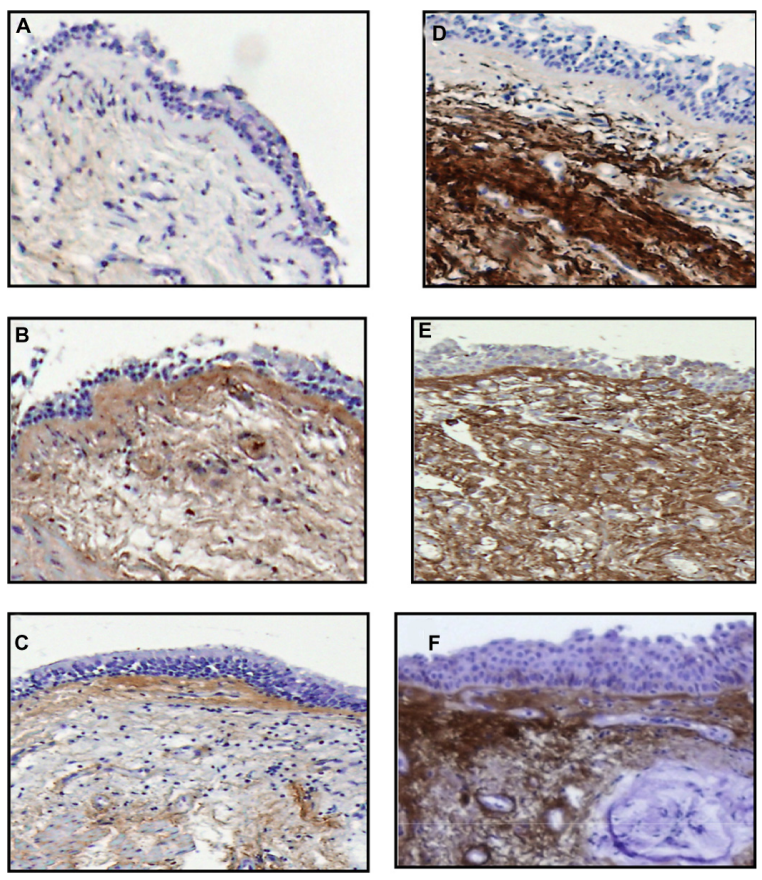

Figure I Immunohistochemical staining for collagen I, versican, and decorin in airway biopsies of control subjects (A-C), and in COPD patients (D-F).

Note: In the biopsy specimens from COPD patients, collagen I, versican, and decorin were prominent within the subepithelial layer.

Abbreviation: COPD, chronic obstructive pulmonary disease. 
the area of the $\mathrm{ASM} / \mathrm{P}_{\mathrm{bm}}{ }^{2}$ in COPD patients versus controls $(P<0.05)$ (Figure 3).

\section{Collagen I and PGs in the smooth muscle layer}

Collagen I and PGs expression in the smooth muscle layer was measured by point counting. An increased protein expression of versican was detected in the smooth muscle layer of COPD patients in comparison with smoker subject controls, but this difference was not statistically significant $(P=0.07)$. No difference was found between the expression of collagen I, decorin, and biglycan in the smooth muscle layer of the two groups.

\section{Correlation of collagen I, PGs, and $\alpha$-SMA with pulmonary function indices}

A significant inverse correlation was observed between $\mathrm{FEV}_{1}$, $\mathrm{FEV}_{1} / \mathrm{FVC}$ ratio, and collagen I expression in the subepithelial layer $\left(r^{2}=0.35, P<0.05\right.$ and $r^{2}=0.51$, and $\left.P<0.01\right)$,

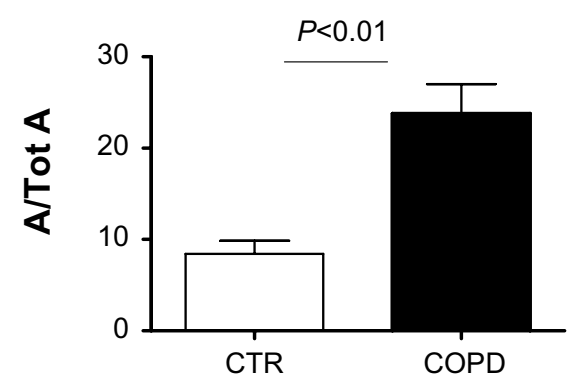

Collagen I

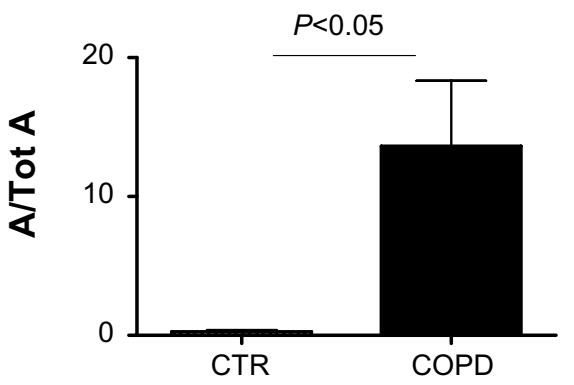

Versican

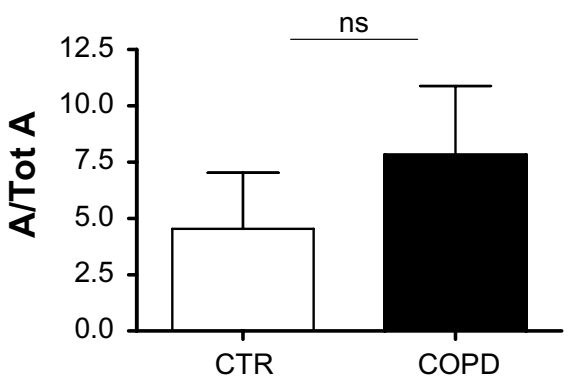

Decorin

Figure 2 Area of positive staining of the proteins in the subepithelial layer standardized for total subepithelial area (A/Tot A) for collagen I, versican, and decorin in biopsy specimens from CTR and COPD subjects.

Note: Data are presented as mean \pm SE.

Abbreviations: CTR, control; COPD, chronic obstructive pulmonary disease; ns, not significant; SE, standard error.

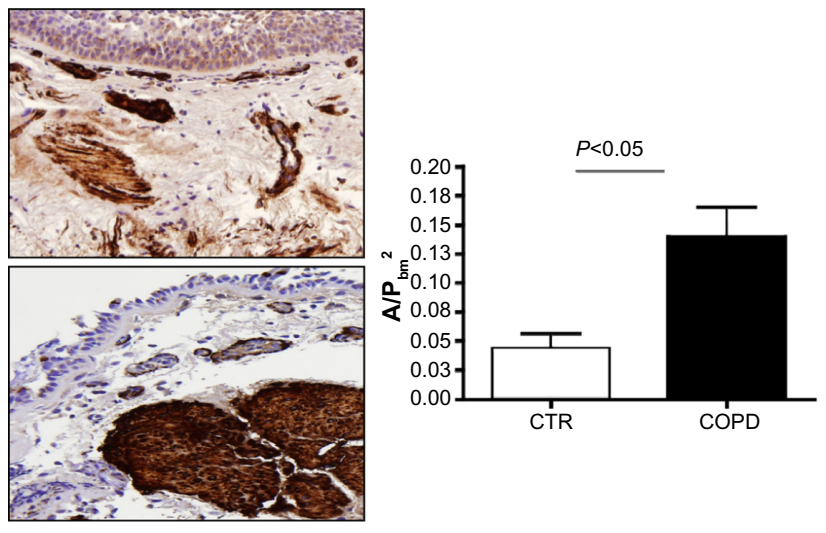

Figure 3 Area of positive staining for $\alpha$-SMA standardized for basement membrane squared $\left(A / \mathrm{P}_{b \mathrm{~b}}{ }^{2}\right)$ in the biopsy specimens from CTR and COPD subjects.

Note: Data are presented as mean \pm SE.

Abbreviations: $\alpha$-SMA, alpha-smooth muscle actin; CTR, control; COPD, chronic obstructive pulmonary disease; SE, standard error.

respectively (Figure 4). No correlation was detected between $\mathrm{FEV}_{1}$ or $\mathrm{FEV}_{1} / \mathrm{FVC}$ ratio and PGs deposition in the subepithelial layer. Although the $\mathrm{FEV}_{1}$ and $\mathrm{FEV}_{1} / \mathrm{FVC}$ ratio tended to be lower in the presence of greater $\alpha$-SMA area, these correlations were not statistically significant.

\section{Discussion}

The results of the present study show that airway wall remodeling affects not only peripheral but also central airways in COPD patients. We actually demonstrated an abnormal deposition of ECM proteins such as collagen I and versican in the subepithelial layer as well as an increase in ASM mass in the large airways of COPD patients compared to control smoker subjects, which partly contributes to their airflow obstruction.

In literature, airway wall remodeling in peripheral airways and its correlation with airflow obstruction has been largely investigated in COPD patients, while few studies have been published on histopathological changes such as subepithelial fibrosis and changes in the ASM in the large airways of these patients.

A potential correlation between inflammation of small airways and airflow obstruction, and wall thickness and smooth muscle mass in large airways was investigated by Tiddens et al. ${ }^{8}$ Their results showed an increase of epithelial and subepithelial layer thickness in the central airways of COPD patients compared to control group, and also an inverse correlation with the $\mathrm{FEV}_{1} / \mathrm{FVC}$ ratio. No significant modifications in the ASM mass within the large airways were documented, but a positive association was found between peripheral airway inflammation and central airway wall 

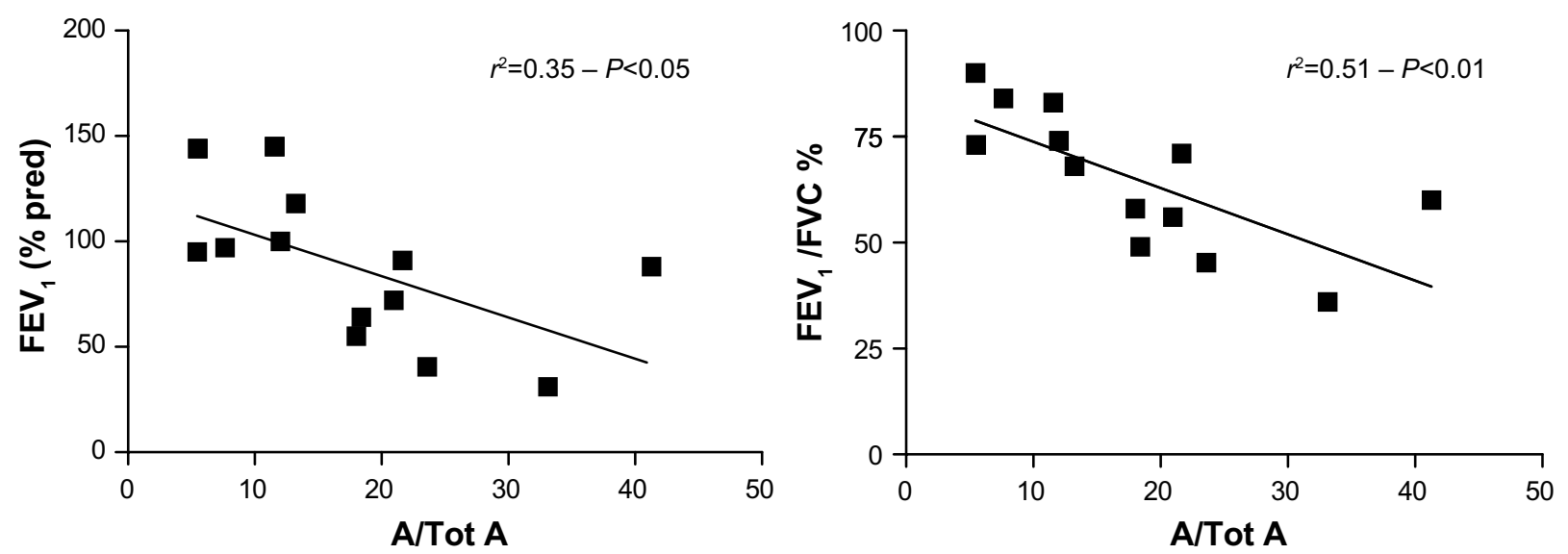

Figure 4 Correlation between spirometric indices and the area of collagen positive staining in the subepithelial area standardized for total subepithelial area (A/Tot A) in control, and COPD subjects.

Note: There is an inverse correlation between obstructive spirometric indices and subepithelial collagen deposition.

Abbreviations: COPD, chronic obstructive pulmonary disease; FEV , forced expiratory volume in the first second; \% pred, \% predicted; FVC, forced vital capacity.

thickness. Such findings led them to speculate that chronic inflammation could induce remodeling in both small, and large airways in COPD patients. ${ }^{8}$ However, they did not investigate the nature of the ECM components involved in the thickening of the wall area of the central airways.

In this study we quantified the subepithelial fibrosis by analyzing different ECM proteins such as collagen type I and PGs (decorin, versican, and biglycan) in an attempt to establish the contribution of these proteins to the remodeling process. In addition, we investigated ASM changes in the central airways of COPD patients.

We found an increased deposition of ECM proteins in the subepithelial layer of the large airways of COPD patients when compared to control smoker subjects. In particular, subepithelial collagen type I and versican deposition was increased in the central airway wall of COPD patients with increased, although not significant, decorin deposition. Our data, reporting an increase in the deposition of the ECM proteins, are in accordance with results from existing literature. ${ }^{8-10}$ It should be noted, however, that Annoni et al recently documented a reduction in type I collagen in the inner layer. ${ }^{11}$ This contrast in findings could be explained by the fact that all the patients enrolled in Annoni et al's study had lung cancer, and neoplasia has documented effects on ECM protein deposition. ${ }^{19}$

Subepithelial collagen deposition was inversely correlated with the $\mathrm{FEV}_{1} / \mathrm{FVC}$ ratio and $\mathrm{FEV}_{1}$, suggesting that protein deposition could play a role in the development of airflow obstruction. Recently, Hasegawa et al demonstrated by computed tomography scan that the airway wall area and luminal area correlate negatively with $\mathrm{FEV}_{1}$ (\% predicted) in both small, and central airways of COPD patients. ${ }^{20}$
Our data endorse the findings published by Kranenburg et al, who found enhanced levels of total collagens I, III, and IV in the surface of the epithelial basement membrane of central airways of COPD patients and a significant inverse correlation between these proteins and $\mathrm{FEV}_{1} \cdot{ }^{10}$ However, the immunostaining approach they used has some limitations. In fact, a visual scoring method was applied to determine protein levels and the assumption was made that staining intensities reflect content. Moreover, the immunostaining quantification was limited at the surface of the epithelial basement membrane.

In our study, we quantified ECM deposition by measuring the area of positive staining, not only at epithelial basement membrane level, but throughout the entire subepithelial layer. Among the ECM proteins we have also included the PGs that, to our knowledge, have never been investigated in the central airway wall of COPD patients.

It is known that PGs are involved in many aspects of matrix biology, including control of tissue hydration (and tissue mechanics as a consequence), regulation of cell adhesion, cell migration and proliferation, interaction with cytokines, and growth factors. For several of these molecules a specific function has been demonstrated: versican for example, being rich of glycosaminoglycan side chains, plays a relevant role in the hydration of the matrix and contributes significantly to tissue viscoelasticity. Decorin and biglycan attach themselves to collagen and influence its fibrillogenesis; they also bind growth factors, such as transforming growth factor beta and fibroblast growth factor, influencing cell proliferation and matrix synthesis. ${ }^{12,16,21}$

We have also studied changes in the ASM area during remodeling on larger airways of COPD subjects. Several 
publications have shown an increase in the ASM in the small airways of COPD patients. .,22-24 $^{2}$

To our knowledge, only Tiddens et al have analyzed the ASM area in the cartilaginous airways of COPD patients without detecting a difference in respect to the control group. ${ }^{8}$ In contrast, our data indicate that the ASM layer is significantly increased in the large airways of patients with COPD, compared to smoker subject controls. It has also been claimed that the increase in the ASM could be correlated with increased matrix deposition in the smooth muscle layer. Although an increase of versican within the ASM bundle in COPD patients was observed, no changes in collagen I, decorin, or biglycan deposition were found when compared with control smoker subjects. Therefore, it seems conceivable that the increase in the ASM layer is mainly due to an enhanced ASM mass itself, and not to an increase in protein deposition in the smooth muscle layer.

In conclusion, the results of the present study show that remodeling also occurs in the central airways of COPD patients where an augmented deposition of ECM proteins and an increase in ASM mass may contribute to airflow obstruction.

Further studies and a greater understanding of changes occurring in the proximal airways are required to establish when remodeling is going to involve central airways in the natural history of COPD and whether early therapeutic interventions might prevent the progression of the remodeling from peripheral to central airways.

\section{Acknowledgment}

This research project was supported by the University of Brescia.

\section{Disclosure}

The authors report no conflicts of interest in this work.

\section{References}

1. Celli BR, MacNee W. Standards for the diagnosis and treatment of patients with COPD: a summary of the ATS/ERS position paper. Eur Respir J. 2004;23(6):932-946.

2. Tam A, Sin DD. Pathobiologic mechanisms of chronic obstructive pulmonary disease. Med Clin North Am. 2012;96(4):681-698.

3. Jeffery PK. Remodeling in asthma and chronic obstructive lung disease. Am J Respir Crit Care Med. 2001;164:S28-S38.
4. Rutgers SR, Timens W, Kauffman HF, Postma DS. Markers of active airway inflammation and remodelling in chronic obstructive pulmonary disease. Clin Exp Allergy. 2001;31(2):193-205.

5. Kuwano K, Bosken CH, Pare PD, Bai TR, Wiggs BR, Hogg JC. Small airways dimensions in asthma and in chronic obstructive pulmonary disease. Am Rev Respir Dis. 1993;148(5):1220-1225.

6. Saetta M, Di Stefano A, Turato G, et al. CD8+ T-lymphocytes in peripheral airways of smokers with chronic obstructive pulmonary disease. Am J Respir Crit Care Med. 1998;157(3 Pt 1):822-826.

7. Hogg JC, Chu F, Utokaparch S, et al. The nature of small-airway obstruction in chronic obstructive pulmonary disease. $N$ Engl J Med. 2004;350:2645-2653.

8. Tiddens HA, Pare PD, Hogg JC, Hop WC, Lambert R, de Jongste JC. Cartilaginous airway dimensions and airflow obstruction in human lungs. Am J Respir Crit Care Med. 1995;152(1):260-266.

9. Liesker JJ, Ten Hacken NH, Zeinstra-Smith M, et al. Reticular basement membrane in asthma and COPD: similar thickness, yet different composition. Int J Chron Obstruct Pulmon Dis. 2009;4:127-135.

10. Kranenburg AR, Willems-Widyastuti A, Moori WJ, et al. Enhanced bronchial expression of extracellular matrix proteins in chronic obstructive pulmonary disease. Am J Clin Pathol. 2006;126:725-735.

11. Annoni R, Lancas T, Tanigawa R, et al. Extracellular matrix composition in COPD. Eur Respir J. 2012;40(6):1362-1373.

12. Iozzo RV. Matrix proteoglycans: from molecular design to cellular function. Annu Rev Biochem. 1998;67:609-652.

13. Rabe KF, Hurd S, Anzueto A, et al. Global strategy for the diagnosis, management, and prevention of chronic obstructive pulmonary disease: GOLD executive summary. Am J Respir Crit Care Med. 2007;176(6): 532-555.

14. Celli BR, Barnes PJ. Exacerbations of chronic obstructive pulmonary disease. Eur Respir J. 2007;29(6):1224-1238.

15. Bolliger CT, Mathur PN, Beamis JF, et al. ERS/ATS statement on interventional pulmonology. Eur Respir J. 2002;19(2):356-373.

16. Pini L, Hamid Q, Shannon J, et al. Differences in proteoglycan deposition in the airways of moderate and severe asthmatics. Eur Respir J. 2007;29(1):71-77.

17. Bolender RP, Hyde DM, Dehoff RT. Lung morphometry: a new generation of tools and experiments for organ, tissue, cell, and molecular biology. Am J Physiol. 1993;265(6 Pt 1):L521-L548.

18. Weibel ER, Hsia CC, Ochs M. How much is there really? Why stereology is essential in lung morphometry. J Appl Physiol. 2007;102(1):459-467.

19. Garusi E, Rossi S, Perris R. Antithetic roles of proteoglycans in cancer. Cell Mol Life Sci. 2012;69:553-579.

20. Hasegawa M, Nasuhara Y, Onodera Y, et al. Airflow limitation and airway dimensions in chronic obstructive pulmonary disease. Am J Respir Crit Care Med. 2006;173(12):1309-1315.

21. Ruoslahti E, Yamaguchi Y. Proteoglycans as modulators of growth factor activities. Cell. 1991;64(5):867-869.

22. Bosken CH, Wiggs BR, Pare PD, Hogg JC. Small airway dimensions in smokers with obstruction to airflow. Am Rev Respir Dis. 1990;142(3): 563-570.

23. Cosio MG, Hale KA, Niewoehner DE. Morphologic and morphometric effects of prolonged cigarette smoking on the small airways. Am Rev Respir Dis. 1980;122(2):265-271.

24. Saetta M, Turato G, Baraldo S, et al. Goblet cell hyperplasia and epithelial inflammation in peripheral airways of smokers with both symptoms of chronic bronchitis and chronic airflow limitation. Am J Respir Crit Care Med. 2000;161(3 Pt 1):1016-1021. 
International Journal of COPD

\section{Publish your work in this journal}

The International Journal of COPD is an international, peer-reviewed journal of therapeutics and pharmacology focusing on concise rapid reporting of clinical studies and reviews in COPD. Special focus is given to the pathophysiological processes underlying the disease, intervention programs, patient focused education, and self management protocols.

\section{Dovepress}

This journal is indexed on PubMed Central, MedLine and CAS. The manuscript management system is completely online and includes a very quick and fair peer-review system, which is all easy to use. Visit http://www.dovepress.com/testimonials.php to read real quotes from published authors.

Submit your manuscript here: http://www.dovepress.com/international-journal-of-copd-journal 